\title{
An Approach for Image Fusion using PCA and Genetic Algorithm
}

\author{
Ramandeep Kaur \\ M.Tech Student \\ Department of Computer Science and Engineering \\ Sri Guru Granth Sahib World University \\ Fatehgarh Sahib
}

\author{
Sukhpreet Kaur \\ Assistant Professor \\ Department of Computer Science and Engineering \\ Sri Guru Granth Sahib World University \\ Fatehgarh Sahib
}

\begin{abstract}
The pattern of mixing multiple images so as to get a single, well developed image is well established. Various fusion methods have been advanced in literature. The current paper is based on image Fusion using PCA and Genetic Algorithm. The pictures of equal size are considered for experimentation. In order to overcome the problems of conventional techniques Genetic Algorithm can be used in collaboration with the technique of PCA (Principal Component Analysis). In Image Fusion, Genetic Algorithm can be signed when optimization of parameter is required. Also for the optimization of the weight values, Genetic algorithm is used. The various parameters used to measure the ability of image fusion technique are Mean Square Error, Entropy, Mean, Bit Error Rate, Mean, Peak Signal to Noise Ratio. From the above experiment we find that this method works well and the quality of the output image is far better than previous methods.
\end{abstract}

\section{General Terms}

Image fusion.

\section{Keywords}

PCA, Genetic algorithm, Mean Square Error, Entropy, Mean, Bit Error Rate, Mean, Peak Signal to Noise Ratio.

\section{INTRODUCTION}

In the $21^{\text {st }}$ century, digital image processing technique has been widely helpful in various occupations[1]. However, it is difficult to produce an image in which all related objects are focused because of the limited depth-of-focus of optical lenses in CCD devices. As a result, the final image will not be in focus everywhere, i.e., if certain object of the scene is in focus, one or another will be out of focus. To eliminate this drawback, a fusion process is needed so that all objects are focused. Image fusion combines conclusive information from various images clicked from the same scene to produce a new fused image. Now this new image will contain the most information. Therefore, the fused image has better characteristics than any of the original images[2]. This final image is more apt for human perception and computerprocessing tasks such as segmentation, feature extraction and object recognition[3]. The methodology of image fusion is being used in various applications like medicine, remote sensing, machine vision, automatic change detection, bio metrics etc[4]. Fusion techniques works on the method of pixel averaging to other methods such as principal component analysis and wavelet transform fusion[5].The major aim of image fusion is to mix the information from various source images of the same scene to output a new final image which provide much more visual information than the source images[6]. Image Fusion increases authenticity, decreases ambiguity and brings down the storage cost by a single effective image than storing multiple images[7][8]. The other benefits of image fusion are wider spatial and temporal coverage, decreased uncertainity, improved reliability and increased robustness of system performance[9]. The present image fusion techniques can be classified into four categories : pixel level, feature level, decision level and symbol level[10][11]. In the end, we can say that two images are mixed in best possible way to reduce the ambiguity and get maximum clarity.

\section{IMAGE FUSION TECHNIQUES}

These techniques having the two input images and these two images are fused with PCA and Genetic algorithm. In the output we get a single image consisting of the major attributes of the both the images.

\subsection{PCA}

It is mathematical method that transforms interrelated variables into the unrelated variable. The verbosity of image can be decreased or eliminated using this technique. The first principal component is fixed along the direction with utmost variance and the second principal component lie in the subspace perpendicular to the first. The third principal component is taken along the direction with utmost variance and in the subspace perpendicular to the first two and so on[13].

\section{Advantages of Principal Component Analysis}

1. This is a spatial domain technique of image fusion, in which works using the pixels. The pixel values are modified to achieve the final results.

2. The property of the PCA of transforming the correlated variable into uncorrelated variables that is used for image fusion.

3. The redundancy of the data is decreased.

4. Large amounts of the inputs are decreased without the actual loss of the information.[9][12][13][14] 
Produce the column vectors of the input images

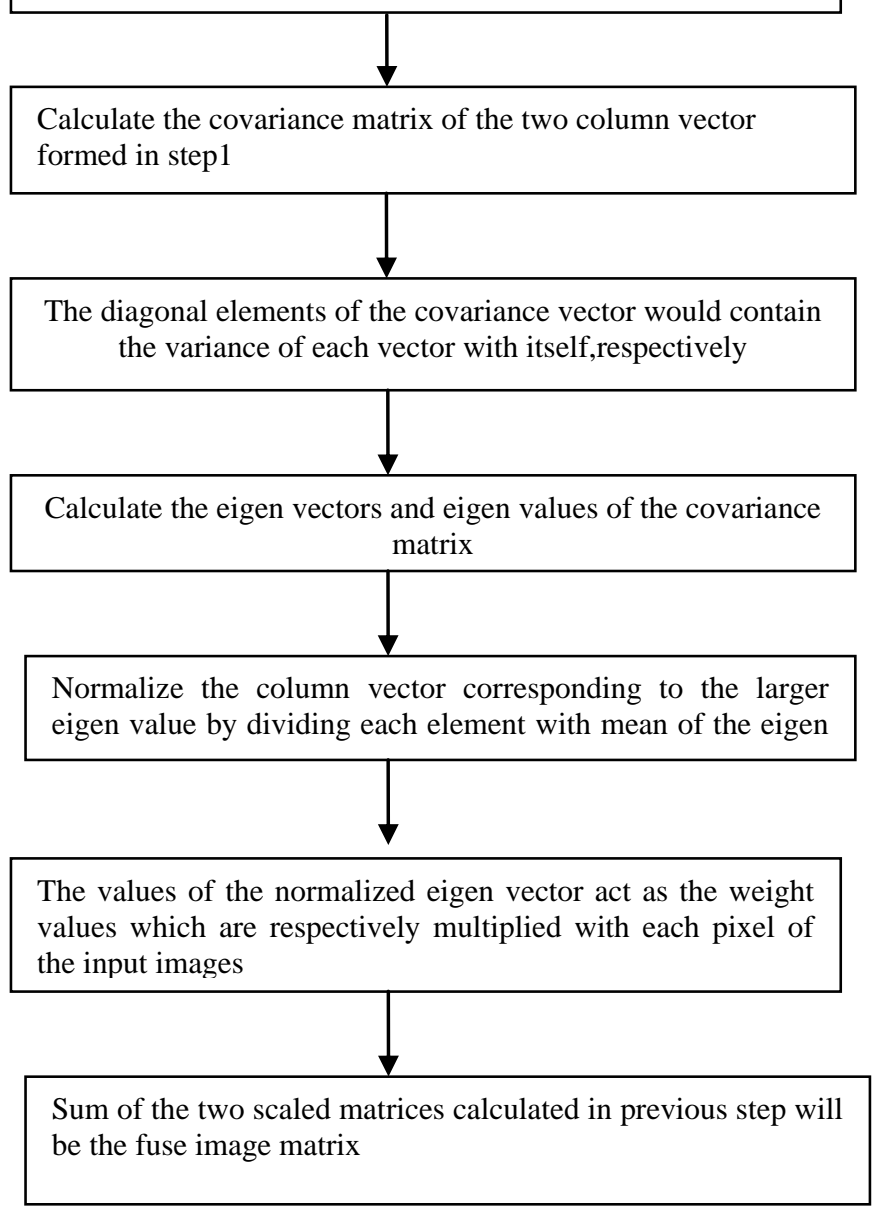

Fig 1:PCA Algorithm

\subsection{Genetic Algorithm-}

Genetic Algorithm is an modern algorithm that uses the guided random search techniques. The GA is designed to imitate processes in natural systems necessary for evolution that follow the Darwin's " survival of the fittest" principle. The genetic algorithm is based on the method of natural selection in which a string of bits is taken as genetic material. The fitness function evaluates each bit. High fitness individuals will take part in coming generation and low fitness bits are eliminated. Next comes the selection step in which there are methods such as tournament selection, truncation selection, roulette wheel selection, rank based selection, steady state selection, elitism and stochastic universal selection. The genetic algorithm has been used in various fields such as function optimization, system identification and control, image processing, robotics, facial recognition, parameter optimization and controllers and multi-objective optimization. In Cao et al. and in Zhang et al., the multifocus image fusion using quality assessment of spatial domain and genetic algorithm is explained. The images are divided into blocks. Each block size is determined using genetic algorithm. The quality assessment can be spatial domain assessment or frequency domain assessment. The image fusion is repetitive process. The GA runs until a final image of greater quality is obtained or maximum numbers of repetitions are executed. GA can also be used for longer duration to get best solution. In medical applications, MRI is considered for the feature elements. Here , optimized feature vector is selected by using genetic algorithm. Das et al. has proposed image fusion scheme in which input images are disintegrated into two subbands as low frequency sub-band and high frequency subband by using wavelet transform. The average data from low frequency sub-bands and maximum information from high frequency sub-bands are selected by applying GA. Finally inverse wavelet transform is used to get fused image. In an approach proposed by Lacewell et al., the GA is used to mix images using the method that combines the pixel level and discrete wavelet transform information. The calculation of fused images done by using the performance parameters such as Mean squared error, Peak signal to noise[17]

1. In Image fusion the GA is used for optimizing the parameters. This method will choose one of the best set of solutions.

2. Genetic algorithms are used in image processing because these are best efficient in solving the large solution space.

3. Genetic algorithms are also used where the intensive search for solution is expensive in terms of computation time.

4. In image fusion the GA is experimented on the obtained features to get the best quality combined image.[15][16]

\section{Advantages of Genetic Algorithm}

1. GA technique will select the best solution and thus solves the problem of multiple solutions.

2. The solution structure and solution parameter problems can be solved at the same time by Structural genetic algorithm.

3. This is the easy and best method with greater clarity and understandability[15][16].

\section{PROPOSED HYBRID MODEL}

1. Initially select two images namely image I and image II from the given set of the data images, so that the fusion is applied on it.

2. After selecting the images apply Principal component analysis on the images to fuse them.

3. Now the initializations of the parameters are done by using Genetic algorithm. This is done in order to obtain the optimized values.

4. After initialization of the parameter by using the genetic algorithm, next step is to generate the population.

5. Now create the fitness function of the population that is generated. This is further used for the calculation of the fitness value.

6. After calculating the fitness value, next step is to perform the cross over and mutation in order to obtain the more optimized fitness value.

7. In this step the value that is obtained by applying the crossover and mutation and the initial fitness value is compared.

8. If the initial value is greater than the new fitness value then save the results.

9. If the value is less than the initial value then go back to step number 7 .

10. Finally the calculation of the parameters are done and the fused image is obtained. 


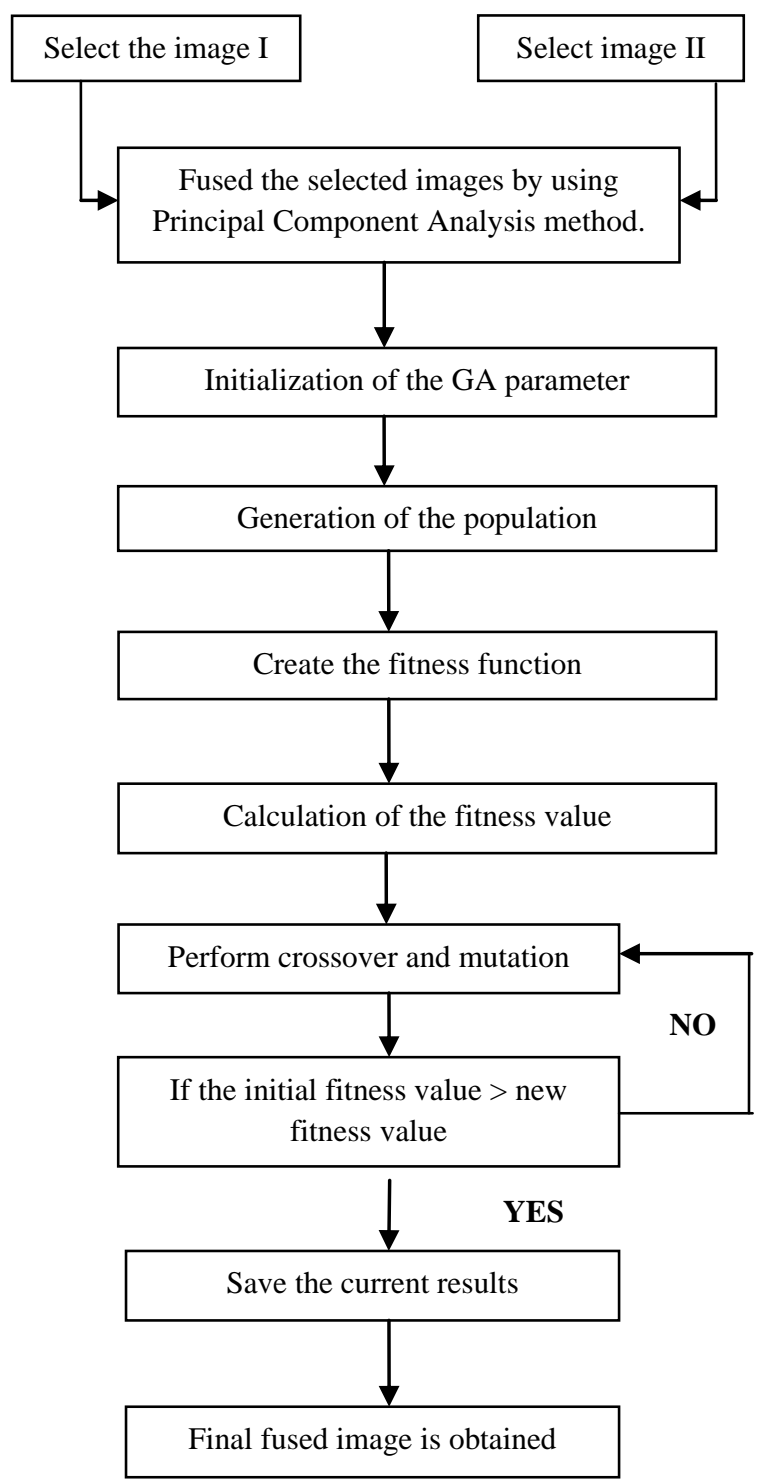

Fig 2: Proposed Hybrid Model

\section{PERFORMANCE ANALYSIS}

There are certain statistical characteristics of the image using which the quality of image fusion can be evaluated; these are entropy, mean, mean square error, bit error rate, peak signal to noise ratio. The average amount of information contained in the number is represented by the size of image entropy, the greater the value, the better the image fusion effect. The various parameters are explained as follows:

1. The probability that the pixel gray level appears is called Entropy. It is an index to calculate the information quantity present in any image. If the measure of entropy increases after fusion, it means that the information increases and the fusion performances are better.

$$
H=-\sum_{\mathrm{i}=0}^{\mathrm{n}} p_{t} \times \log _{2}\left(p_{t}\right)
$$

2. Mean is the Characterization of the average image of the entire pixel values of the image, the greater the mean value, the greater the average brightness of the image, $I(i, j)$ of the image $(i, j)$ of the pixel values.

$$
\bar{I}=\frac{1}{M \times N} \sum_{i=0}^{M-1} \sum_{j=0}^{N-1} I(i, j)
$$

3. PSNR, The value of PSNR will be high when the fused and original images are similar. Higher value indicates better fusion.

$$
\operatorname{PSNR}(d B)=20 \log \frac{\sqrt[255]{3 M N}}{\sqrt{\left.\sum_{i=1}^{M} \sum_{j=1}^{N}(i, j)-B(i, j)\right)^{2}}}
$$

$\mathrm{B}$ is the perfect fused image, $\mathrm{i}$ is the index of rows , j is the index of columns.

4. MSE differentiates the two images. If it has lower value, it means fused and reference images are similar and better performance.

$$
M S E=\frac{1}{m n} \sum_{i=1}^{m} \sum_{j=1}^{n}\left(A_{i j}-B_{i j}\right)^{2}
$$

A - the perfect image, B - the fused image to be assessed, $\mathrm{i}$ - pixel row index, $\mathrm{j}-$ pixel column index, $\mathrm{m}, \mathrm{n}$ - No. of row and column

5. BER is the reciprocal of PSNR. It implies the amount of error between the original image and fused image.

$$
B E R=\frac{1}{P S N R}
$$

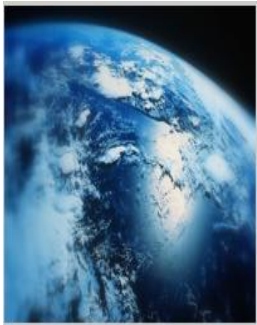

Left side blur

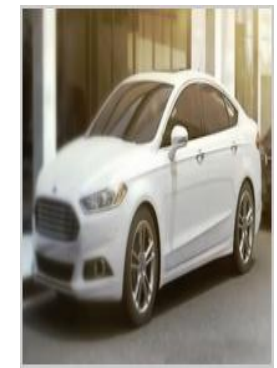

Left side blur

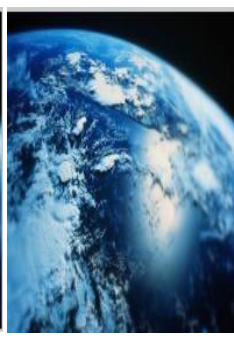

Right side blur

Fig3: Image1

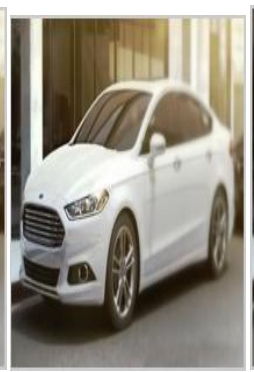

Right side blur

Fig4: Image2

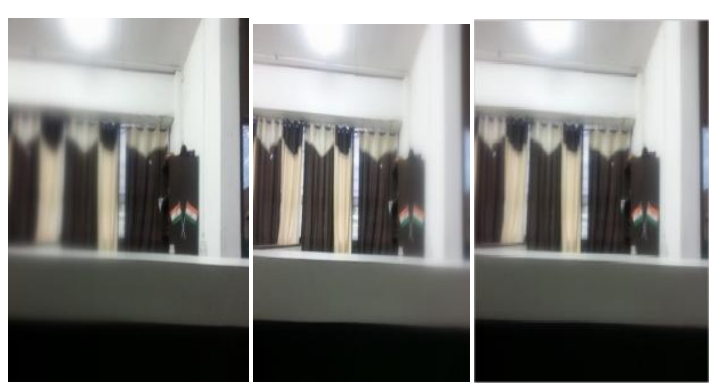

Left side blur Right side blur Final fused imageFig5:

Image 3

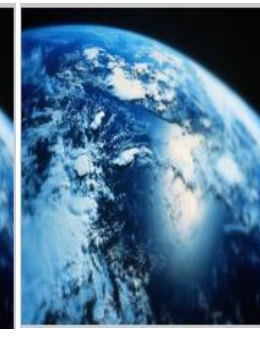

Final fused image

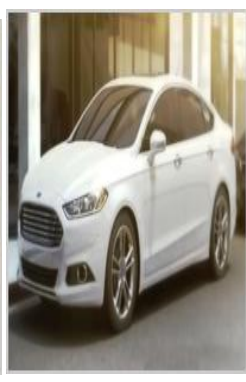

Final fused image 

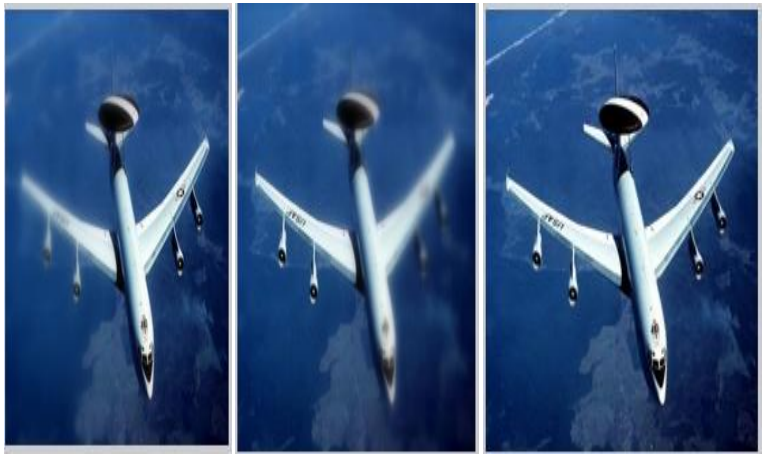

Left side blur Right side blur Final fused imageFig6: Image4

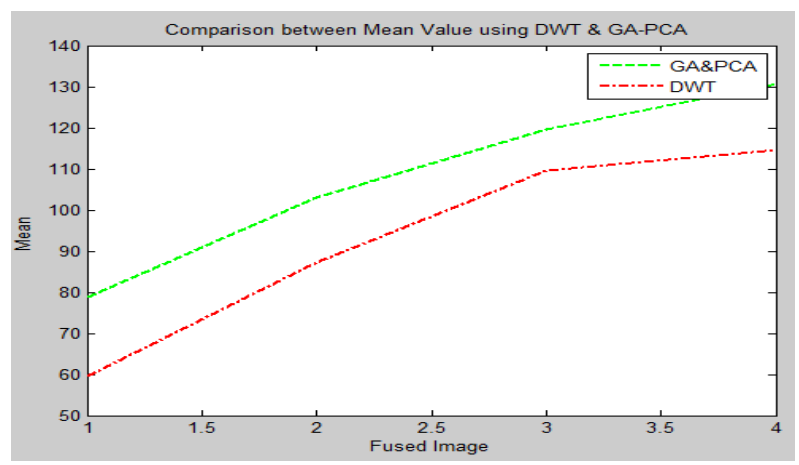

Fig7: Mean

Table 1: Mean of 4 images

\begin{tabular}{|c|c|c|}
\hline & $\begin{array}{c}\text { Results using } \\
\text { DWT }\end{array}$ & $\begin{array}{c}\text { Results using GA } \\
\text { and PCA }\end{array}$ \\
\hline Image1 & 87.3258 & 103.0964 \\
\hline Image2 & 114.6719 & 130.62 \\
\hline Image3 & 109.49 & 119.6260 \\
\hline Image4 & 59.7323 & 78.7770 \\
\hline
\end{tabular}

Table 1 presents the results obtained while testing the algorithm with respect to Mean.From the result projected,it is clear that the proposed method shows the significant improvement in terms of Mean when it is compared with traditional technique.

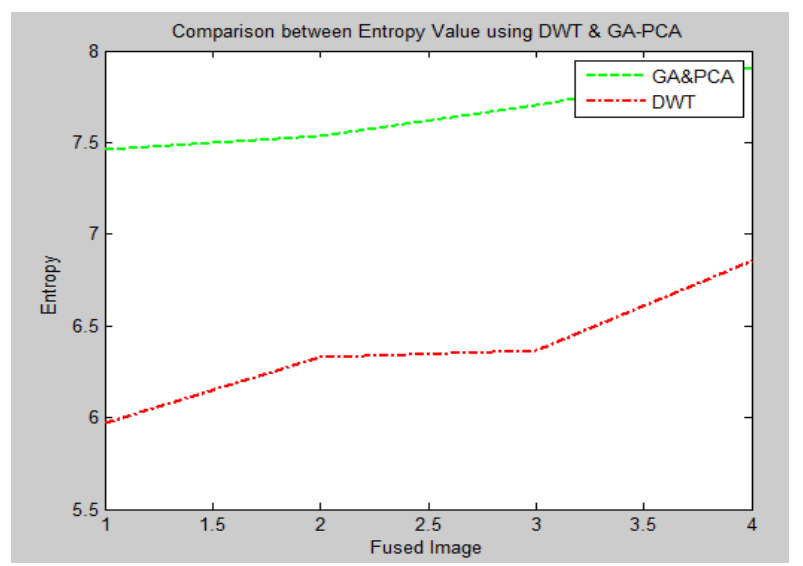

Fig8: Entropy
Table 2: Entropy of 4 images

\begin{tabular}{|c|c|c|}
\hline & $\begin{array}{c}\text { Results using } \\
\text { DWT }\end{array}$ & $\begin{array}{c}\text { Results using GA } \\
\text { and PCA }\end{array}$ \\
\hline Image1 & 6.3298 & 7.7052 \\
\hline Image2 & 6.8547 & 7.9051 \\
\hline Image3 & 5.9690 & 7.4603 \\
\hline Image4 & 6.3675 & 7.5347 \\
\hline
\end{tabular}

Table 2 presents the results obtained while testing the algorithm with respect to Entropy.From the result obtained,it is clear that the proposed method shows the significant improvement in terms of Entropy when it is compared with traditional technique.

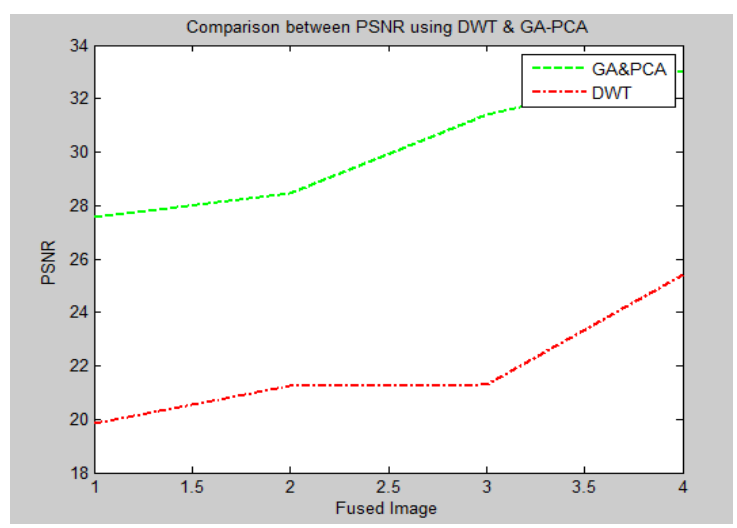

Fig 9: PSNR

Table 3: PSNR of 4 images

\begin{tabular}{|c|c|c|}
\hline & $\begin{array}{c}\text { Results using } \\
\text { DWT }\end{array}$ & $\begin{array}{c}\text { Results using GA } \\
\text { and PCA }\end{array}$ \\
\hline Image1 & 21.2793 & 27.5619 \\
\hline Image2 & 21.2724 & 31.4252 \\
\hline Image3 & 25.3931 & 28.4682 \\
\hline Image4 & 19.8627 & 33.0310 \\
\hline
\end{tabular}

Table 3 presents the results obtained while testing the algorithm with respect to PSNR.From the result obtained,it is clear that the proposed method shows the significant improvement in terms of PSNR when it is compared with traditional technique.

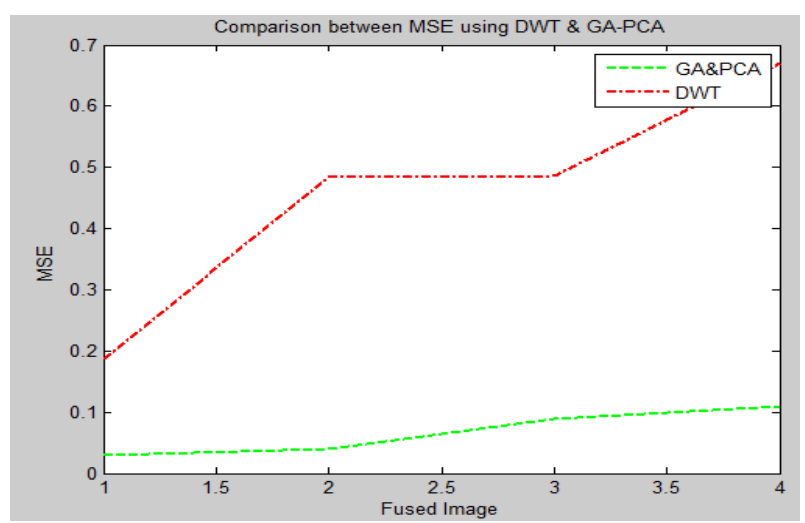

Fig 10: MSE 
Table 4: MSE of 4 images

\begin{tabular}{|l|l|l|}
\hline & $\begin{array}{l}\text { Results using } \\
\text { DWT }\end{array}$ & $\begin{array}{l}\text { Results using GA } \\
\text { and PCA }\end{array}$ \\
\hline Image1 & 0.4843 & 0.1140 \\
\hline Image2 & 0.4851 & 0.468 \\
\hline Image3 & 0.1878 & 0.0925 \\
\hline Image4 & 0.6711 & 0.0324 \\
\hline
\end{tabular}

Table 4 presents the results obtained while testing the algorithm with respect to MSE.From the result obtained,it is clear that the proposed method shows the significant improvement in terms of MSE when it is compared with traditional technique.

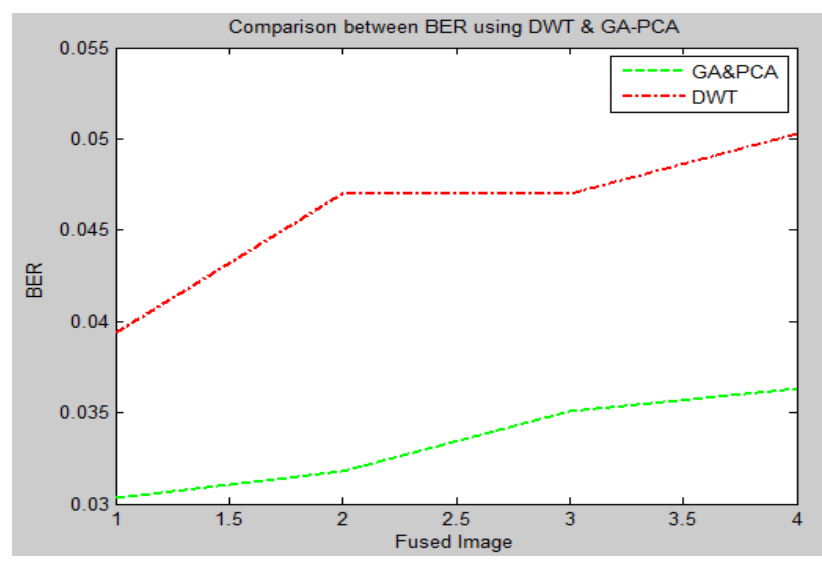

Fig 11: BER

Table 5: BER of 4 images

\begin{tabular}{|c|c|c|}
\hline & $\begin{array}{c}\text { Results using } \\
\text { DWT }\end{array}$ & $\begin{array}{c}\text { Results using GA } \\
\text { and PCA }\end{array}$ \\
\hline Image1 & 0.0470 & 0.0363 \\
\hline Image2 & 0.0470 & 0.0318 \\
\hline Image3 & 0.0394 & 0.0351 \\
\hline Image4 & 0.0503 & 0.0303 \\
\hline
\end{tabular}

Table 5 presents the results obtained while testing the algorithm with respect to BER.From the result obtained,it is clear that the proposed method shows the significant improvement in terms of BER when it is compared traditional technique.

\section{CONCLUSIONS}

Method implemented for PCA and genetic algorithm is gives reliable fused image than original image for finding the quality of the image taken parameters was Mean Square Error, ,Bit Error Rate of fused image reduce both kind of error and increases the Entropy, Mean, Peak Signal to Noise Ratio. In lot of applications image fusion is used to obtain the high quality image from two source images. Experimental results are obtained by using the hybrid approach. Based on the results obtained from experiments it can be seen that the proposed method provides a good results. Image fusion using PCA and genetic algorithm has been simulated using MATLAB.

\section{F UTURE WORK}

The traditional image fusion have drawbacks and do not meet the needs of fusion. This can be eliminated by using the hybrid systems. After obtaining the results it is analyzed that the above proposed technique is far better than the traditional techniques. Hybrid techniques are more efficient techniques than the traditional techniques. Therefore in the future work we focus on improving conventional system by employing the hybrid system.

\section{REFERENCES}

[1] R. C. Gonzalez and R. E. Woods Digital Image Processing, 2nd edition, Pearson Education, 2004.

[2] C. Pohl, "Review Article Multisensor Image Fusion in Remote Sensing: Concepts, Methods and Applications", International Journal of Remote Sensing, 2010,Vol. 19,No. 5,pp. 823-854.

[3] Wencheng Wang, "A Multi-focus Image Fusion Method Based on Laplacian Pyramid", Journal of Computers,2011, Vol. 6, pp. 2259-2566.

[4] S.M. Mukane, Y. S. Ghodake, and P. S. Khandagle, "Image Enhancement Using Fusion by Wavelet Transform and Laplacian Pyramid.", arXiv preprint arXiv:1401.6129,2013.

[5] Pavithra C, “ Fusion of Two Images Based on Wavelet Transform”, International Journal of Innovative Research in Science, Engineering and Technology,2013, Vol. 2, Issue 5, pp. 1814-1819.

[6] Mentor, $\mathrm{Ph}$ D. Student, and Hariharasudhan Viswanathan, "Image Fusion Using Laplacian Pyramid Transform".

[7] Kusum Rani, " Study of Image Fusion using Discrete wavelet and Multiwavelet Transform", International Journal of Innovative Research in Computer and Communication Engineering,2013. Vol. 1, Issue 4, pp. 95-99.

[8] Gagandeep Kaur, "A New Hybrid Wavelet Based Approach for Image Fusion", International Journal of Innovative Research in Science, Engineering and Technology,2015, Vol. 4, Issue 1, pp. 1934- 1043.

[9] Reham Gharbia, "Image fusion techniques in remote sensing”, arXiv preprint arXiv:1403.5473,2014.

[10] Sukhpreet Singh and Rachna Rajput, "Multiple Image Fusion Using Laplacian Pyramid", International journal of Engineering And Computer Science,2014,Vol 3,Issue 12,pp 9442-9446.

[11] S.M. Mukane, Y. S. Ghodake, and P. S. Khandagle, "Image enhancement using fusion by wavelet transform and laplacian pyramid", arXiv preprint arXiv:1401.6129,2013.

[12] Metwalli, M.R, "Image Fusion Based on Principal Component Analysis and High-pass Filter", Computer Engineering \& Systems. ICCES International Conference on,2009, pp. 63-70.

[13] V.P.S. Naidu and J.R. Raol, "Pixel-level Image Fusion using Wavelets and Principal Component Analysis", Defenses Science Journal,2008, Vol. 58, No. 3, pp. 338352. 
[14] Nirosha Joshitha, "Image Fusion using PCA in Multifeature Based Palmprint Recognition", International Journal of Soft Computing and Engineering (IJSCE),2012, VoL.02, Issue-2, pp 226-230.

[15] Gehad Mohamed Taher, "Image fusion approach with noise reduction using Genetic Algorithm", (IJACSA) International Journal of Advanced Computer Science and Applications, 2013,Vol. 4, No. 11.

[16] Jun Kong, "Multi-focus Image Fusion Using Spatial
Frequency and Genetic Algorithm”, IJCSNS:International Journal of Computer Science and Network Security,2008, Vol. .8,No. 2.

[17] Jyoti S.Kulkarni, "A Survey of Image Fusion using Genetic Algorithm", International Journal of Computer Applications (0975 - 8887) National Conference on Advances in Computing,2015. 